\title{
An Influential Recommendation System Usage for General Users
}

\author{
Nikhat Akhtar \\ Research Scholar Ph.D (Computer Science \& \\ Engineering) M.Tech, B.Tech (Computer Science \\ \& Engineering) \\ Department of Computer Science \& Engineering, \\ Babu Banarasi Das University, Lucknow, India
}

\author{
Devendera Agarwal, $\mathrm{PhD}$ \\ Director, BBDNIIT, \\ Lucknow, India
}

\begin{abstract}
Recommender systems are extensively seen as an effective means to combat information overload, as they redound us both narrow down the number of items to choose. They are seen as assistance us make better decisions at a lower transaction cost. Hence, recommender systems have become omnipresent in e-commerce and are also increasingly used in services in different other domains both online and offline where the number of items exceeds our potentiality to consider them all individually. The research papers recommender systems are software applications or systems that help individual users to discover the most relevant research papers to their needs. These systems use filtering techniques to create recommendations. These techniques are categorized majorly into collaborative-based filtering, content-based technique, and hybrid algorithm. In addition, they assist in decision making by providing product information both personalized and non-personalized, summarizing community opinion, search research papers, and providing community critiques. As a result, recommender systems have been shown to ameliorate the decision.
\end{abstract}

\section{Keywords}

Recommendations System, Tagging, Information Retrieval, E-Commerce, Collaborative Filtering.

\section{INTRODUCTION}

The Recommender systems have become enormously common in recent years. They are applied in a miscellaneous of applications. The most popular ones are probably movies, books, news, music, research papers, social tags, and products in general [1]. A Recommender system based on the past ratings of a user recommendation on one of the three waysthrough collaborative-filtering technique, content-based technique, and hybrid algorithm [2]. Recommender systems assistance in two partially overlapping ways, firstly by helping us discover items that are likely to liking us, generally by suggesting items and secondly, by helping us decide which item(s) as well as research papers to choose, many times by providing information, e.g. community opinion and critiques [3]. The primary uses of recommender systems is to assist us in making better decisions. A recommender system collects precedence data from its users and then aggregates this information for its users. The recommender systems research community has embraced both the systems that recommend items to users, many times in the form of lists of suggested items that are personalized to the user, and the systems that help users evaluate items, therefore covering systems that endow recommendations, that endow prediction, and that endow community opinion [3]. This definition covers a great variety of recommender systems, ranging from automated and personalized systems that technologists mostly see as the best or, infrequently, as the only proper approach to recommender systems to non-personalized ones that are presented the same way to all the users of the system [4]. Later researchers are spending an amount of time on internet in researching papers that cover their interest due to the high volume of accessible resources online. Many online scientific papers repository such as journals and conference proceedings, arrange their published research papers according to the year of publication, volumes and numbers, which make it arduous to discover related papers. This means that to discover papers, a reader or researcher has to know the link to the journals and then one can search them by year of publication, volumes and numbers which consumes a large amount of time [5]. The most reliable solution for this problem is paper recommendation systems. The paper's recommendation systems purpose of recommending episodic papers to researchers with respect to their particular demands [6].

\section{RELATED WORK}

The first look at the prime definition by Resnick and Varian [7] who coined the term recommender system in the first place Neumann, [8] They saw the term recommender system as being more usual than the phrase collaborative filtering that was coined by Goldberg et al. [9], the developers of the first recommender system, and that had been widely adopted in the literature [7]. Resnick and Varian [7] saw recommender systems as a means to "assist and augment" the "natural social process" of using the recommendations of others to make choices when we do not have adequate personal knowledge or experience of the alternatives. In a typical recommender system, user "recommendations" is aggregated and directed to appropriate recipients. In certain cases, the aggregation represents the primary transformation and, in others, the value comes from matching "the recommenders" with those seeking recommendations. Resnick and Varian [7] placed recommender systems in a technical design space that is defined by five dimensions firstly recommendation/ evaluation content (e.g. A rating or a mentioning of an item), secondly explicit/ implicit entry, thirdly anonymity level of the recommendation, fourthly aggregation approach, and fifthly use of recommendations.

To summarize the definition by Resnick and Varian [7] along the three dimensions, firstly recommender systems can display personalized or non personalized output (i.e. recommendations), as displaying textual annotations with the evaluated item is non-personal if the same set is displayed to all users [10], secondly recommender systems can be algorithmic or non-algorithmic, as presenting textual annotations as they were entered is non-algorithmic while 
employing collaborative filtering is clearly an algorithmic approach; and thirdly since textual evaluations may be displayed together with the item, recommender systems can both help users discover items of interest from the multitude of items and help them decide between items and research papers.

An improved example of one such definition is that of Knijnenburg et al. [11] that states unequivocally that the algorithm is an essential part of any recommender system and that the algorithm provides personalized recommendations, i.e. offer every user a personalized subset of the items, tailored to the user's and researcher preferences, typically as an ordered list of recommendations. Correspondingly, many see personalization as inherent to recommenders; e.g. Pommeranz et al. [12] see recommender systems as tools that endow personalized recommendations to people, Buder and Schwind [13] state that recommender systems are personalized, i.e. they propose items that are adaptively tailored to the needs, interests, and preferences of a user, and Cremonesi et al. [14] consider the objective of a recommender system to be to predict which items a user will discover interesting or useful. Wang, et al., [15] proposed a collaborative topic regression model which harmonizes ideas from $\mathrm{CF}$ and content analysis based on probabilistic topic modeling.

Next Cristiano, et al., [16] introduced a source independent framework for the research paper recommendation. Their method be in need of as input only a single research paper and generates several potential queries by using terms in that paper, which are then submitted to existing web information sources that hold research papers. We feel that all researcher institutions are subscribed to those well known Web information sources providing full papers such as the ACM Digital Library, IEEE Xplore, and Science Direct, etc., and using the full candidate paper can renovate the accuracy and provide relevant papers. Jeckmans et al. [17] maintain that a recommender system provides a set of items that is most relevant to a particular user of the system, and Campochiaro et al. [18] write that recommender systems provide users with a short list of items which they are certain to enjoy.

\section{RECOMMENDER SYSTEMS AND TAGGING}

The tagging can be seen as build up a recommender system. The tags, user contributed free form, non-hierarchical labels that function as metadata, have traditionally been applied in information systems in general for social navigation remembering, discovery, and sharing items and information structuring and organizing items as well as research papers and information and serendipitous browsing [19]. The collaborative tagging systems are in use in numerous services , e.g. Last.fm, Del.icio.us, Flickr.com, and Amazon.com. Tags and folksonomies that come out from tagging have been used in recommender systems in sundry ways and in sundry roles, as have recommender systems being used to ameliorate tagging systems, too [20]. In effect, recommender systems have been used to recommend tags and tags have been used to enhance recommendation [20]. Tag recommendation systems help users discover better tags and they have previously been used since the preliminary days of tagging systems Del.icio.us, founded in 2003, for example, had previously incorporated a tag recommender in 2005 [21]. As long as tag recommenders assist users in the tagging process, they are also used to raise the opportunity of getting an item and research papers annotated and importantly, to consolidate the tagging vocabulary [22]. The tags have also widely been used in the recommendation process, typically as additional knowledge [20]. For example, tagging data have been used to identify alike users or viewed as additional information about the items to be recommended. Tags have been used to enhance both content-based recommender systems (e.g. using tags as item descriptions) and collaborative filtering recommender systems, e.g. using tags to discover tantamount users in addition to hybrid systems [19]. Through exploiting existing interaction between users, items and tags, such approaches have managed to renovate algorithmic ascendancy both in terms of predictive accuracy and coverage. Concretely, tags have been used to enrich recommender systems in recent years in different ways and the trend seems to continue to produce advancement.

\section{RECOMMENDER SYSTEMS VS INFORMATION RETRIEVAL SYSTEMS}

The recommender systems are decision support systems in that they are information systems, they are used in decision making, and they are meant to support humans, not to replace them [23]. The recommender systems are different from customary decision-support systems based on various aspects. The customary decision-support systems were aimed at managers or analysts that used such systems for assistance in different tasks, generally in planning tasks and consequently such systems generally employed process models. The users of recommender systems generally face a class of arduous known as preferential preference problems, and hence, recommender systems employ preference models and support the integration of decision criteria across alternatives [23]. Recommender systems also share alike characteristics with knowledge based systems, as they necessity to explain their reasoning to the users in order to engender trust in themselves and their recommendations. The prospective users may have suspicions about the benevolence of the systems in addition to having suspicions about their capability, and, consequently, engendering trust on, the user is a key factor in recommender system acceptance [24].

In the end recommender systems are not information retrieval systems. When a recommender system returns recommendations, they are unequivocal to be items that are worthy of consideration, i.e. likely to be interesting or useful [25]. Opposed to the items an information retrieval system returns are unequivocal to be matched to the query a search engine returns all matching items and research papers, ranking them by the degree of the match. The dissimilarity between search engines and recommender

systems by stating that while search engines help us discover what we know we are questing for, serendipitous discovery that recommender systems offer helps us discover the rest [26]. In a consciousness, a recommender system functions as an information agent for the user, often providing individualized recommendations that are episodic and zestful to the user.

\section{SOCIAL NAVIGATION OF RECOMMENDER SYSTEMS}

Recommender systems also represent a type of social navigation [27]. The term social navigation was renumber by Dourish and Chalmers [28] who saw it as "moving from one item to another" that is induced as an artifact of the activity of one more or a group of others. Social navigation permission 
people to benefit from the presence and activity of others. The concept of social navigation is based on the observation that watching and socialize with other people is part of everyday information seeking behavior. Accordingly, the consideration that people tend to use social interaction with other people as a means of deficiency uncertainty when selecting items was behind the concept of recommender systems; e.g. people frequently ask other people for recommendations when prefers a movie. Both are largely about people in a virtual community impacting each other as if socialized without actually socialize, although social navigation of recommender systems does also include more direct means of assisting in navigation, e.g. recommendations of links by email [29]. Social navigation systems exploit social practices and behavior of assistance users navigate and discover by making the collective, aggregate, or individual behavior visible and advantageous as a basis for making decisions.

In addition to the social texture, including ratings, reviews, tags, and more algorithmic recommender systems being advantageous in discovering and selecting salient items and making the place more alive and inviting, it can also provide social affordance awareness of what behavior is convenient and what is not. The social navigation of recommender systems, can transform the environment and behavior in it not only by assisting us in discovering items of interest and making decisions but also by engendering social rules that affect behavior. Ultimately, social navigation of recommender systems is not simply about helping users navigate more proficiently, or helping them discover items and research papers of interest speedily.

\section{USES IN E-COMMERCE}

The recommender systems started to take place in academia, e-commerce sites, research papers also saw their potential and started to implement them to help their users discover items of interest out of the huge number of items and research papers available [30]. The recommender systems were transshipped from novelties used by a few E-commerce sites, to serious business tools that are re-shaping the world of E-commerce. Today, scenario recommender systems are embedded in an extensive range of commerce and content applications (both online and offline) and the scope of recommender systems has expanded from collaborative filtering to numerous approaches [30]. Alternately, online retailers such as Amazon.com opted for the item based (or item-item) approaches that have faster response times, in particular if the item relationships are precomputed offline as relationships between items tend to be rather stable, and that actually tend to be slightly more accurate, too. The limitation

of e-commerce also includes such social media services as Twitter, LinkedIn and Facebook and also recommender systems play a vital role there too, as such services use them in multiple ways, e.g. recommending news in addition to recommending believable friends [31].

\section{BENEFITS OF RECOMMENDER SYSTEMS}

In this paper recommender systems can be seen as being beneficial both to service providers and users. For users, recommender systems lower the transaction costs of discover and selecting items as well as research papers, be it in an online shopping environment or library. For e-commerce players, recommender systems can enhance revenues, as they are progressively seen as an effective means to sell more products. Afterwards recommender systems offer user support possibilities that can enable them to become digital information centers by allowing them to move beyond catalog searches and research papers and to support dissimilar user needs.

Literally, recommender systems suggest items and research papers to users and then help users decide which item(s) and research papers to select for purchasing, viewing, downloading, and so on. The purpose of recommender systems is to reduce transaction costs, i.e. to enable users to select the item and research papers with the lowest overall transaction cost. In order to discover and select an item and research papers, a user must go through a transaction process that starts with a search for relevant items is followed by a comparison stage that involves in e-commerce. Recommender systems do induce behavioral transformation by enhancing the lift factor and the metamorphose rate, and by diversifying purchases and orienting them towards the long tail. Therewith, recommendations are inherently persuasive in that they are discern as a correct answer by default users have some vested trust in recommender systems [32].

\section{CONCLUSION}

The recommender systems are endowing users with the essential confidence to make decisions by deficiency uncertainty. Concretely, recommender systems are the prime exhibit of social navigation that we have today. In addition to helping users discover items and research papers of interest based on the behavior and preferences of the user community and providing community consideration to assist in decision making, recommender systems also have a vital role in providing social texture. The recommendation system, recent interests focuses on increasing precision of recommendation. In other words, allow personalized of customized information is wanted and an inevitability of new marketing strategy, such as web personalization, one-to-one marketing, and customer relationship management is an exigency in the social practice working area and academic research. In this paper, we are discussing Tagging, uses in E-Commerce, recommender systems vs Information retrieval systems, social navigation of recommender systems and benefits of recommender systems. Recommendation systems for research articles are advantageous applications, which for illustration help researchers keep track of their research field and recommend episodic papers with respect to their individual interests. The recommender systems assist us in cognitively demanding tasks, e.g. by deficiency the complexity of searching for items and research papers of interest with item and research paper's suggestions. Finally, the most apparent pursuit of a recommender system is to propitiate its users' information exigency.

\section{REFERENCES}

[1] P. Gupta, A. Goel, J. Lin, A. Sharma, D.Wang, and R. B. Zadeh WTF; The who-to-follow system at Twitter, Proceedings of the 22nd international conference on World Wide Web, pp 505-514, 13 May 2013.

[2] H. Jafarkarimi, A.T.H. Sim and R. Saadatdoost A Naïve Recommendation Model for Large Databases, International Journal of Information and Educa tion Technology, June 2012.

[3] Schafer, J. B., Konstan, J. A., \& Riedl, J. (2001). ECommerce recommendation applications. Data Mining and Knowledge Discovery, 5(1-2), 115-153. doi:10.1023/A:1009804230409 
[4] Lam, S. K. T., Frankowski, D., \& Riedl, J. (2006). Do you trust your recommendations? An exploration of security and privacy issues in recommender systems. In G. Müller (Ed.), Proceedings of the 2006 International Conference on Emerging Trends in Information and Communication Security (ETRICS'06), LNCS 3995, 1429. Berlin Heidelberg, Germany: Springer. doi:10.1007/11766155_2.

[5] Nikhat Akhtar, Prof. (Dr.) Devendera Agarwal, "A Literature Review of Empirical Studies of Recommendation Systems" International Journal of Applied Information Systems (IJAIS) USA, Volume 10, No. 2, Pages 6 - 14, December 2015, ISSN 2249 - 0868, Link http://www.ijais.org/archives/volume10/number2/ 839-2015451467, DOI : 10.5120/ijais2015451467.

[6] Y. Liang, Q. Li and T. Qian, "Finding Relevant Papers Based on Citation Relations", Springer-Verlag Berlin Heidelberg, (2011), pp. 403-414.

[7] Resnick, P., \& Varian, H. R. (1997). Recommender systems. Communications of the ACM, 40(3), 56-58. doi:10.1145/245108.245121

[8] Neumann, A. W. (2007). Motivating and supporting user interaction with recommender systems. In L. Kovács, N. Fuhr, \& C. Meghini (Eds.), Proceedings of the 11th European Conference on Research and Advanced Technology for Digital Libraries (ECDL'07), LNCS 4675, 428-439. Berlin Heidelberg, Germany: Springer. doi:10.1007/978-3-540-74851-9_36.

[9] Goldberg, D., Nichols, D., Oki, B. M., \& Terry, D. (1992). Using collaborative filtering to weave an information tapestry. Communications of the ACM, 35(12), 61-70. doi:10.1145/138859.138867.

[10] Schafer, J.B., Frankowski, D., Herlocker, J., \& Sen, S. (2007). Collaborative filtering recommender systems. In P. Brusilovsky, A. Kobsa, \& W. Nejdl (Eds.), The Adaptive Web, LNCS 4321, 291-324. Berlin Heidelberg, Germany: Springer. doi:10.1007/978-3-540-72079-9_9.

[11] Knijnenburg, B. P., Willemsen, M. C., Gantner, Z., Soncu, H., \& Newell, C. (2012). Explaining the user experience of recommender systems. User Modeling and User-Adapted Interaction, 22(4-5), 441504.doi:10.1007/s11257-011-9118-4.

[12] Pommeranz, A., Broekens, J., Wiggers, P., Brinkman, W.-P., \& Jonker, C. M. (2012). Designing interfaces for explicit preference elicitation: a user-centered investigation of preference representation and elicitation process. User Modeling and User-Adapted Interaction, 22(4-5), 357-397. doi:10.1007/s11257-011-9116-6.

[13] Buder, J., \& Schwind, C. (2012). Learning with personalized recommender systems: A psychological view. Computers in Human Behavior, 28(1), 207-216. doi:10.1016/j.chb.2011.09.002.

[14] Cremonesi, P., Turrin, R., Lentini, E., \& Matteucci, M. (2008). An evaluation methodology for collaborative recommender systems. In Proceedings of the 2008 International Conference on Automated Solutions for Cross Media Content and Multi-channel Distribution (AXMEDIS '08), 224-231. Washington, DC, USA: IEEE Computer Society.doi:10.1109/AXMEDIS.2008.13.
[15] C. Wang and D. M. Blei, "Collaborative Topic Modeling for Recommending Scientific Articles", In Proc. of the 17th ACM SIGKDD International Conference on Knowledge Discovery and Data Mining, (2011), pp. 448-456.

[16] C. Nascimento, A. H. F. Laender, A. S. da Silva and M. A. Gonçalves, "A Source Independent Framework for Research Paper Recommendation". ACM, (2011) June 13-17, Ottawa, Ontario, Canada.

[17] Jeckmans, A. J. P., Beye, M., Erkin, Z., Hartel, P., Lagendijk, R. L., \& Tang, Q. (2013). Privacy in recommender systems. N. Ramzan, R. van Zwol, J.-S. Lee, K. Clüver, \& X.-S. Hua (Eds.), Social Media Retrieval, 263-281. London, UK: Springer. doi:10.1007/978-1-4471-4555-4_12.

[18] Campochiaro, E., Casatta, R., Cremonesi, P., \& Turrin, R. (2009). Do metrics make recommender algorithms? In Proceedings of the 2009 International Conference on Advanced Information Networking and Applications Workshops (WAINA '09), 648-653. Washington, DC, USA: IEEE Computer Society. doi:10.1109/WAINA.2009.127.

[19] Gedikli, F., \& Jannach, D. (2010). Rating items by rating tags. In Proceedings of the 2nd Workshop on Recommender Systems and the Social Web (RSWEB'10), 25-32.

[20] Bobadilla, J., Ortega, F., Hernando, A., \& Gutiérrez, A. (2013). Recommender systems survey. KnowledgeBased Systems, 46, 109132.doi:10.1016/j.knosys.2013.03. 012.

[21] Jäschke, R., Marinho, L., Hotho, A., Schmidt-Thieme, L., \& Stumme, G. (2007). Tag recommendations in folksonomies. In J. N. Kok, J. Koronacki, R. Lopez De Mantaras, S. Matwin, D. Mladenič, \& A. Skowron (Eds.), Proceedings of the 11th European Conference on Principles and Practice of Knowledge Discovery in Databases (PKDD 2007), LNCS 4702, 506-514. Berlin Heidelberg, Germany: Springer. doi:10.1007/978- 3-54074976-9_52.

[22] Konstan, J. A. (2012). Recommender Systems: The Power of Personalizatio Q\&A. http://learning.acm.org/webinar/recommender_qa.cfm.

[23] Xiao, B., \& Benbasat, I. (2007). E-commerce product recommendation agents: Use, characteristics, and impact. MIS Quarterly, 31(1), 137-209. http://www.misq.org/ecommerce-product-recommendation-agentsusecharacteristics-and-impact.html.

[24] Knijnenburg, B. P., Reijmer, N. J. M., \& Willemsen, M. C. (2011). Each to his own: How different users call for different interaction methods in recommender systems. In Proceedings of the fifth ACM conference on Recommender systems (RecSys '11), 141-148. New York, NY, USA: ACM., doi:10.1145/2043932.2043960.

[25] Burke, R. (2007). Hybrid web recommender systems. In P. Brusilovsky, A. Kobsa, \& W. Nejdl (Eds.), The Adaptive Web, LNCS 4321, 377-408. Berlin Heidelberg, Germany: Springer. doi:10.1007/978-3-540-720799_12.

[26] Hangartner, R. (2007). What is the recommender industry? Mobile Groove.com 
http://www.mobilegroove.com/guest-columnwhat-is-therecommender-industry- 750 .

[27] Schafer, J.B., Frankowski, D., Herlocker, J., \& Sen, S. (2007). Collaborative filtering recommender systems. In P. Brusilovsky, A. Kobsa, \& W. Nejdl (Eds.), The Adaptive Web, LNCS 4321, 291-324. Berlin Heidelberg, Germany: Springer. doi:10.1007/978-3-540-72079-9_9.

[28] Dourish, P., \& Chalmers, M. (1994). Running out of space: Models of information navigation. Short paper presented at HCI'94, two pages. http://www.dcs.gla.ac.uk/ matthew/papers/hci94.pdf.

[29] Dieberger, A. (1997). Supporting social navigation on the World Wide Web. International Journal of HumanComputer Studies, 46(6), 805-825. doi:10.1006/ijhc.1996.0111.
[30] Konstan, J. A., \& Riedl, J. (2012). Recommender systems: From algorithms to user experience. User Modeling and User-Adapted Interaction, 22, 101- 123. doi:10.1007/s11257-011-9112-x.

[31] Gupta, P., Goel, A., Lin, J., Sharma, A., Wang, D., \& Zadeh, R. (2013). WTF: The who to follow service at Twitter. In Proceedings of the $22^{\text {nd }}$ International Conference on World Wide Web (WWW '13), 505-514. Republic and Canton of Geneva, Switzerland: International World Wide Web Conferences Steering Committee. http://www2013.org/proceedings/p505.pdf.

[32] Adomavicius, G., Bockstedt, J., Curley, S., \& Zhang, J. (2013)," Do recommender systems manipulate consumer preferences? A study of anchoring effects," SSRN. doi:10.2139/ssrn.2285042 